\title{
Metabolomics analysis reveals an effect of homocysteine on arachidonic acid and linoleic acid metabolism pathway
}

\author{
BIN LI $^{1 *}$, GUANGQIANG GAO $^{1 *}$, WANYING ZHANG ${ }^{1}$, BOWEN LI $^{1}$, CHUN YANG $^{1}$, \\ XIAOFENG JIANG ${ }^{1}$, YAPING TIAN ${ }^{2}$ and HONGYAN LIANG ${ }^{1}$
}

\author{
${ }^{1}$ Department of Clinical Laboratory, The Fourth Affiliated Hospital of Harbin Medical University, Harbin, \\ Heilongjiang $150001{ }^{2}$ Department of Clinical Biochemistry, Chinese PLA General Hospital, Beijing 100853, P.R. China
}

Received August 24, 2016; Accepted May 16, 2017

DOI: $10.3892 / \mathrm{mmr} .2018 .8643$

\begin{abstract}
An increase in serum homocysteine level has been associated with an increased risk of vascular disease; however, the biochemical mechanisms that underlie these effects remain largely unknown. The present study aimed to use high-performance liquid chromatography-mass spectrometry (HPLC-MS) to demonstrate the effects of serum homocysteine on human blood metabolites. A total of 75 fasting serum samples were investigated in the present study. Using a threshold of $15 \mu \mathrm{mol} / 1$ serum homocysteine level, samples were divided into high- and low-homocysteine groups, and the serum extracts were analyzed with an HPLC-MS-based method. A total of 269 features exhibited significant differences and correlation with serum homocysteine levels in the electrospray ionization-positive [ESI(+)] mode, and 69 features were identified in the ESI(-) mode between the two groups. The principal component analysis plot revealed a separation between the high- and the low-homocysteine groups. Metabolite set enrichment analysis identified arachidonic acid metabolism and linoleic acid metabolism as the two pathways with significantly enriched differences. These results revealed that arachidonic acid and linoleic acid metabolism may be associated with serum homocysteine levels and may be involved in homocysteine-induced vascular disease.
\end{abstract}

\section{Introduction}

The non-protein $\alpha$-amino acid homocysteine is an intermediate product that occurs during the normal biosynthesis of cysteine

Correspondence to: Professor Hongyan Liang, Department of Clinical Laboratory, The Fourth Affiliated Hospital of Harbin Medical University, 37 Yiyuan Street, Harbin, Heilongjiang 150001, P.R. China

E-mail: aileenliang2005@163.com

*Contributed equally

Key words: homocysteine, metabolomics, high-performance liquid chromatography-mass spectrometry, arachidonic acid, linoleic acid and methionine. Hyperhomocysteinemia has been proposed to be an independent risk factor for cardiovascular disease (1). Several previous studies have reported that an increase in the level of serum homocysteine may be involved in the pathogenesis of vascular diseases, such as coronary artery disease (2), coronary heart disease $(3,4)$, atherosclerotic vascular disease $(5,6)$, stroke $(7,8)$ and ischemic heart disease $(9)$. A meta-analysis reported that for every $5 \mu \mathrm{mol}$ increase in serum homocysteine level the danger of coronary heart disease increased by $60-80 \%$ in adults (10); compared with healthy subjects, there was a sevenfold increase in the mortality rate in patients with high levels of serum homocysteine (11). A recent report indicated that an elevation in serum homocysteine levels may lead to vascular disease in the general population (12).

Although it has long been considered that an increased serum homocysteine level was associated with an increased risk of vascular disease, the biochemical mechanisms that underlie these effects in serum remain unclear. The present study aimed to investigate the effects of serum homocysteine levels on human blood metabolites in humans. To avoid any potential influences on the results, the samples in which vascular diseases were identified were not included in the present study.

Metabolomics analyses have previously been demonstrated to provide a dynamic depiction of metabolic status (13), and have been successfully applied in several fields of research, including disease diagnosis (14), biomarker screening $(15,16)$ and in characterizing biological pathways $(17,18)$. Therefore, the present study used a high-performance liquid chromatography-mass spectrometry (HPLC-MS)-based metabolomics approach to detect the biochemical alterations associated with homocysteine levels in human serum.

\section{Materials and methods}

Serum sample collection and preparation. The present study was approved by the Ethics Committee of the Fourth Affiliated Hospital of Harbin Medical University (Harbin, China). All participating subjects were informed of their rights and written informed consent was obtained. A total of 161 fasting blood samples were obtained from patients aged 25-79 years during routine physical examination at the Department of Physical Examination Center of the Fourth Affiliated Hospital 
of Harbin Medical University between April 5 and April 18, 2015. The samples were divided into high- and low-serum homocysteine groups based on a threshold of $15.0 \mu \mathrm{mol} / \mathrm{l}$ serum homocysteine level. Patients were questioned about their lifestyles, including smoking status (non-smokers were identified as having never smoked or stopped smoking $>1$ year ago) and alcohol intake (non-drinkers were identified as having not consumed alcohol in the previous 2 weeks and having not consumed $>38 \%$ alcohol v/v and $>200 \mathrm{ml}$ in the previous month). Patients were also asked whether they had already been diagnosed with vascular disease. In order to prevent vascular disease and unhealthy habits affecting the results of the present study, patients adhering to the following criteria were excluded from the study: Hypertension, diabetes, hyperlipemia, obesity, coronary heart disease, atherosclerosis, stroke, cerebral embolism and recipients of folic acid and/or vitamin B12 supplements. Hypertension was diagnosed when the systolic blood pressure (SBP) was $\geq 140 \mathrm{mmHg}$ and/or diastolic blood pressure (DBP) was $\geq 90 \mathrm{mmHg}$. Diabetes was diagnosed when the fasting plasma glucose level was $\geq 6.1 \mathrm{mmol} / 1$. Hypercholesterolemia and hyperlipemia were defined as total cholesterol $\geq 6.22 \mathrm{mmol} / \mathrm{l}$ and triglycerides $\geq 2.26 \mathrm{mmol} / \mathrm{l}$, respectively, according to the 2007 Dyslipidemia Prevention Guide in Chinese Adults (19). Obesity was defined as having a body mass index $(\mathrm{BMI}) \geq 28.00 \mathrm{~kg} / \mathrm{m}^{2}$, according to the 2006 Guidelines for Prevention and Control of Obesity in Chinese Adults (20). Coronary heart disease was confirmed by coronary angiography. Stroke, atherosclerosis and cerebral embolism were diagnosed by computed tomography scanning. A total of 86 samples were excluded and 75 samples were retained for further investigation.

All fasting blood samples were centrifuged at 1,408 x g for 5 min immediately at room temperature, then the serum was collected. Levels of fasting serum glucose (FSG), total cholesterol (TC), high-density lipoprotein cholesterol (HDL-C), low-density lipoprotein cholesterol (LDL-C), triglyceride (TG) and serum homocysteine were determined using Hitachi 7600 Automatic Biochemistry Analyzer (Hitachi Instrument Service, Tokyo, Japan). All reagents and calibrators were purchased from Roche Diagnostics (Basel, Switzerland). All quality controls (serum homocysteine, FSG, TC, HDL-C, LDL-C and TG) were purchased from Bio-Rad Laboratories, Inc. (Hercules, CA, USA). Hyperhomocysteinemia was defined as having an abnormally high level ( $>15 \mu \mathrm{mol} / \mathrm{l})$ of homocysteine in the serum sample (21).

Each serum sample was used for metabolite extraction prior to HPLC-MS analysis. Briefly, acetonitrile (400 $\mu \mathrm{l}$; Chromasolv chromatographic grade; Sigma-Aldrich; Merck KGaA, Darmstadt, Germany) was added to the serum (200 $\mu \mathrm{l})$, and the mixture was vortexed for $1 \mathrm{~min}$ and incubated at room temperature for $10 \mathrm{~min}$, followed by centrifugation at $18,500 \mathrm{x} \mathrm{g}$ at room temperature for $10 \mathrm{~min}$. The supernatant $(400 \mu \mathrm{l})$ was removed and completely evaporated using ultra-high-purity $(99.9 \%)$ nitrogen gas in water bath at $40^{\circ} \mathrm{C}(22)$, and $100 \mu 1$ of a mixture of acetonitrile and water (3:1) was added into each tube. The solution was passed through a syringe filter $(0.22 \mu \mathrm{m})$ into a $2 \mathrm{ml}$ glass vial prior to HPLC-MS analysis.

Metabolomics profiling with ESI. HPLC-MS analysis was performed with a Shimadzu HPLC System (Shimadzu
Corporation, Kyoto, Japan) coupled to an AB Sciex API4000+ mass spectrometer with electrospray ionization (ESI) in the positive (+) and negative (-) modes. The sample solution $(5 \mu \mathrm{l})$ was injected into an HPLC InertSustain C18 Column ( $2.1 \times 150 \mathrm{~mm} ; 3 \mu \mathrm{m}$; GL Sciences Inc., Torrance CA, USA). The flow rate of the mobile phase was $0.35 \mathrm{ml} / \mathrm{min}$, and analytes were eluted from the column under a gradient (solvent $\mathrm{A}, 0.1 \%$ formic acid in water; solvent B, $0.1 \%$ formic acid in acetonitrile). The optimal conditions for HPLC separation and ESI detection are shown in Table I.

Data and statistical analysis. Raw data was in an instrument specific format (.wiff) and were converted to common data format (.mzXML) format, using the Wiff to mzXML translator software (version 1.3; Applied Biosystems; Thermo Fisher Scientific, Inc.). The program XCMS was used for nonlinear alignment of the raw data (.mzXML files) in the time domain and automatic integration and extraction of the peak intensities (23). Accurate masses of features that were identified as significantly different and correlated with serum homocysteine levels were searched against the Metlin databases. MetaboAnalyst 3.0 software $(24,25)$ was used for multivariate statistical calculations and plotting and metabolic pathway enrichment analysis (26). Differences between high- and low-serum homocysteine groups were determined using a two-tailed Student's t-test with 5\% false discovery rate (FDR). Correlations between the level of serum homocysteine and accurate masses of features were analyzed using Pearson's correlation coefficient. Serum biochemical indicators are presented as the mean \pm standard deviation. FDR-adjusted $\mathrm{P}<0.05$ was considered to indicate a statistically significant difference. These analyses were performed using R Bioconductor (version 2.15.3; http:www.r-project.org/). The experimental workflow for the metabolomics studies performed is depicted in Fig. 1.

\section{Results}

The mean values of serum homocysteine concentration in the high- and low-serum homocysteine groups were 29.4 and $11.9 \mu \mathrm{mol} / 1$, respectively. The clinical biochemical characteristics of the samples are summarized in Table II. No significant differences were identified between the two groups for the following characteristics: Sex, age, FSG, TC, HDL-C, LDL-C, DBP, SBP and BMI.

A total of 75 serum samples were analyzed by HPLC coupled to ESI(+)- or ESI(-)-MS. Raw data from the individual analyses were subjected to nonlinear data alignment. Univariate statistics were used to screen the differential features between the high- and low-serum homocysteine groups, and multivariate statistics were used to determine group separation. In the ESI(+) mode, a total of 1,949 features were detected, of which $695(35.7 \%)$ exhibited significant differences between the two groups (FDR-adjusted $\mathrm{P}<0.05$; Table III). Correlation analysis revealed that $269(13.8 \%)$ of the 1,949 different features are correlated with the level of serum homocysteine (FDR <0.05), ranging from 0.41 to 0.65 for 59 positive correlation features and -0.68 to -0.41 for 210 negative correlation features. In the ESI(-) mode, a total of 1,721 features were detected, of which 157 (9.1\%) exhibited significant differences between 
Table I. HPLC separation and ESI-mass spectrometry detection conditions.

\begin{tabular}{ll}
\hline Component & \\
\hline HPLC InertSustain C18 Column & $150 \times 2.1 \mathrm{~mm} ; 3 \mu \mathrm{m}$ \\
Mobile phase A & $0.1 \% \mathrm{HCOOH}$ in $\mathrm{H}_{2} \mathrm{O}$ \\
Mobile phase B & $0.1 \% \mathrm{HCOOH}$ in $\mathrm{CH}_{3} \mathrm{CN}$ \\
Gradient elution & $\mathrm{B} \%=2 \%$ maintained $(0-2 \mathrm{~min})$, increased to $20 \%$ in 4 min, $70 \%$ linearly increased (4- 8 min), \\
& $100 \%$ in a further 4 min, and $100 \%$ maintained 2 min $(14-18$ min), followed by \\
& re-equilibration to the initial conditions in 6 min $(18-25$ min $)$ \\
Flow rate & $0.35 \mathrm{ml} / \mathrm{min}$ \\
Injection volume & $5 \mu 1$ \\
Polarity & $\mathrm{ESI}(+)$ and ESI(-) \\
Gas1 & $15 \mathrm{l} / \mathrm{h}$ \\
Curtain gas & $10 \mathrm{l} / \mathrm{h}$ \\
DP & $45 \mathrm{~V}$ \\
Source temperature & $375^{\circ} \mathrm{C}$ \\
MS range & $\mathrm{m} / \mathrm{z} 40-1,500$
\end{tabular}

ESI, electrospray ionization; HPLC, high-performance liquid chromatography.

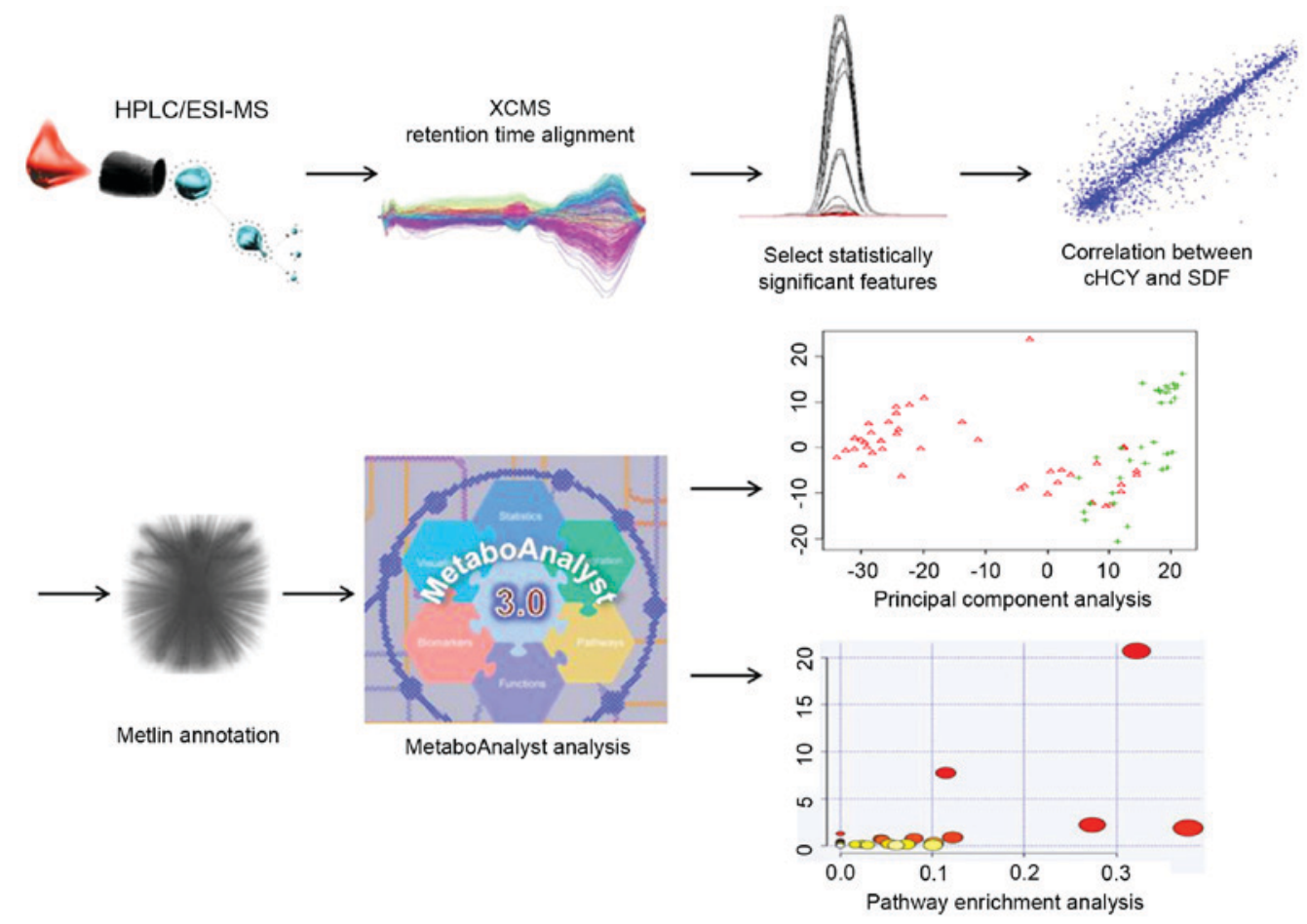

Figure 1. Experimental workflow for the serum metabolomics of different homocysteine level in human. Serum samples were detected with ESI in the positive and negative modes. The program XCMS was used for nonlinear alignment of raw data and the extraction of peak intensities. The SDF between high- and low-serum homocysteine groups were selected based on 75 serum samples and following this, the correlation between the SDF values and serum homocysteine levels were analyzed. The different and correlated features selected were searched against the Metlin database, and the corresponding compounds that were matched in the Metlin database were further analyzed by principal component analysis and metabolic pathway enrichment analysis methods in MetaboAnalyst software. cHCY, homocysteine concentration; HPLC/ESI-MS, high-performance liquid chromatography/electrospray ionization-mass spectrometry; SDF, significantly different features.

the two groups (FDR <0.05), and a total of $69(4.0 \%)$ of the 1,721 features had clear correlations with the level of serum homocysteine (FDR <0.05), ranging from 0.40 to 0.68 for 32 positive correlation features and -0.63 to -0.39 for 37 negative correlation features. Matlin database searches revealed that only 36 accurate masses that represented significant differences and correlated features were matched (Table IV), and the corresponding 77 compounds were selected.

Principal component analysis of the 36 features aforementioned (that is, plotting principal component 1 
Table II. Biochemical characteristics in the high- and low-serum HCY groups.

\begin{tabular}{|c|c|c|c|}
\hline Characteristic & High-HCY group & Low-HCY group & P-value \\
\hline Number & 33 & 42 & \\
\hline Sex (female/male) & $15 / 18$ & $20 / 22$ & 0.69 \\
\hline Age, year & $47 \pm 10.7$ & $44 \pm 8.6$ & 0.25 \\
\hline $\mathrm{FSG}, \mathrm{mmol} / 1$ & $5.5 \pm 1.4$ & $5.5 \pm 1.3$ & 0.86 \\
\hline $\mathrm{TC}, \mathrm{mmol} / \mathrm{l}$ & $4.9 \pm 0.8$ & $4.7 \pm 0.9$ & 0.12 \\
\hline HDL-C, mmol/l & $1.3 \pm 0.4$ & $1.3 \pm 0.3$ & 0.63 \\
\hline LDL-C, mmol/l & $3.2 \pm 0.7$ & $3.0 \pm 0.6$ & 0.14 \\
\hline $\mathrm{TG}, \mathrm{mmol} / \mathrm{l}$ & $1.91 \pm 0.5$ & $1.78 \pm 0.7$ & 0.43 \\
\hline $\mathrm{DBP}, \mathrm{mmHg}$ & $81 \pm 5.3$ & $79 \pm 7.1$ & 0.16 \\
\hline $\mathrm{SBP}, \mathrm{mmHg}$ & $131 \pm 9.5$ & $128 \pm 6.7$ & 0.22 \\
\hline BMI, $\mathrm{kg} / \mathrm{m}^{2}$ & $27.2 \pm 4.9$ & $28.3 \pm 5.2$ & 0.58 \\
\hline
\end{tabular}

Data are presented as the mean \pm standard deviation; $\mathrm{P}<0.05$ were considered to indicate a statistically significant difference. BMI, body mass index; DBP, diastolic blood pressure; FSG, fasting serum glucose; HCY, serum homocysteine; HDL-C, high-density lipoprotein cholesterol; LDL-C, low-density lipoprotein cholesterol; SBP, systolic blood pressure; TC, total cholesterol; TG, triglyceride.

Table III. Summary of the metabolomics profiles using two different MS-based methods.

\begin{tabular}{lccc}
\hline Method & Total peaks & Significant changed peaks $(\%)$ & Significant changed and correlated peaks $(\%)$ \\
\hline HPLC/ESI(+)-MS & 1,949 & $695(35.7)$ & $269(13.8)$ \\
HPLC/ESI(-)-MS & 1,721 & $157(9.1)$ & $69(4.0)$ \\
\hline
\end{tabular}

HPLC/ESI(+/-)-MS, high-performance liquid chromatography/electrospray ionization-mass spectrometry in the positive or negative mode, respectively.
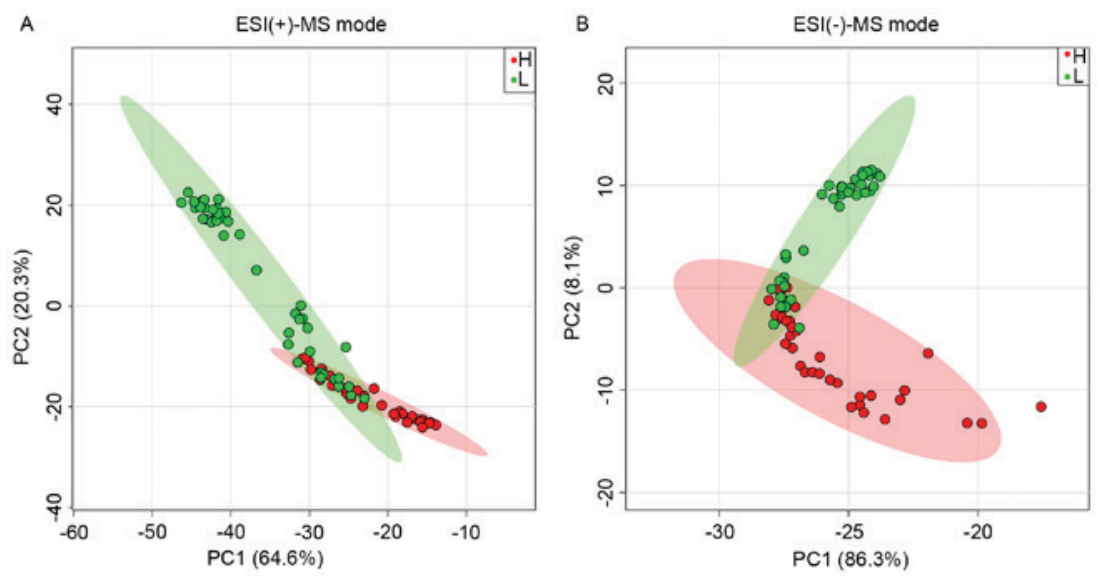

Figure 2. PCA of the high- and low-serum homocysteine groups in ESI(+)-MS and ESI(-)-MS modes. PCA plots were generated from serum sample data sets of two groups, high- vs. low-serum homocysteine. (A) PCA plot of the two sample groups in ESI(+)-MS mode. (B) PCA plot of the two sample groups in ESI(-)-MS mode. Green circles represent the low-serum homocysteine group; Red circles indicate the high serum homocysteine group. ESI(+/-)-MS, electrospray ionization-mass spectrometry in the positive/negative mode; PCA, principal component analysis.

vs. principal component 2) revealed a separation of the highand the low-serum homocysteine sample groups (Fig. 2). In the ESI(+)-MS mode, the mean value of the samples in the low-level group was $10.8 \mu \mathrm{mol} / 1$ compared with that of the high-level groups $(39.1 \mu \mathrm{mol} / \mathrm{l})$ in the non-close-knit area (Fig. 2A). However, a portion of the samples in one group was dispersed to the other group in close-knit areas and exhibited similar levels of serum homocysteine: The mean value of the low-level samples in the close-knit area was $14.3 \mu \mathrm{mol} / \mathrm{l}$, compared with the high-level samples (16.6 $\mu \mathrm{mol} / \mathrm{l})$. Similar separation was found in the ESI(-)-MS mode (Fig. 2B). In the ESI(-)-MS mode, the mean value of the samples in the low-level group was $11.2 \mu \mathrm{mol} / 1$ compared with that of the high-level groups $(36.8 \mu \mathrm{mol} / \mathrm{l})$ in the non-close-knit area (Fig. 2B). 
Table IV. Detailed information of 36 accurate masses.

\begin{tabular}{|c|c|c|c|c|}
\hline Feature & $\mathrm{FC}$ & P-value1 & Cor & P-value2 \\
\hline M110T182 & 7.42 & $2.19 \times 10^{-07}$ & 0.64 & $6.19 \times 10^{-07}$ \\
\hline M305T140 & 1.50 & $9.70 \times 10^{-08}$ & -0.54 & $6.44 \times 10^{-05}$ \\
\hline M311T139 & 1.53 & $9.25 \times 10^{-09}$ & -0.49 & $4.90 \times 10^{-04}$ \\
\hline M313T131 & 1.45 & $1.19 \times 10^{-06}$ & -0.58 & $1.10 \times 10^{-05}$ \\
\hline M315T127 & 1.53 & $5.81 \times 10^{-06}$ & -0.52 & $1.76 \times 10^{-04}$ \\
\hline M317T125 & 1.68 & $1.37 \times 10^{-08}$ & -0.52 & $1.52 \times 10^{-04}$ \\
\hline M321T139 & 1.70 & $4.77 \times 10^{-05}$ & -0.48 & $7.44 \times 10^{-0}$ \\
\hline M335T120 & 1.46 & $5.84 \times 10^{-06}$ & -0.59 & $6.44 \times 10^{-06}$ \\
\hline M367T135 & 1.62 & $5.49 \times 10^{-08}$ & -0.52 & $1.65 \times 10^{-04}$ \\
\hline M373T128 & 1.27 & $2.71 \times 10^{-04}$ & -0.57 & $2.40 \times 10^{-05}$ \\
\hline M385T122 & 1.41 & $7.76 \times 10^{-04}$ & -0.54 & $7.55 \times 10^{-05}$ \\
\hline M418T138 & 1.31 & $2.39 \times 10^{-05}$ & -0.50 & $4.16 \times 10^{-04}$ \\
\hline M423T137 & 1.25 & $3.53 \times 10^{-05}$ & -0.57 & $1.98 \times 10^{-05}$ \\
\hline M427T139 & 1.42 & $8.16 \times 10^{-06}$ & -0.49 & $4.68 \times 10^{-04}$ \\
\hline M429T132 & 1.50 & $9.97 \times 10^{-08}$ & -0.51 & $2.30 \times 10^{-04}$ \\
\hline M438T135 & 1.30 & $1.86 \times 10^{-05}$ & -0.62 & $1.76 \times 10^{-06}$ \\
\hline M441T118 & 1.55 & $2.61 \times 10^{-07}$ & -0.61 & $2.41 \times 10^{-06}$ \\
\hline M445T126 & 1.38 & $3.56 \times 10^{-04}$ & -0.60 & $4.56 \times 10^{-06}$ \\
\hline M451T121 & 1.64 & $3.33 \times 10^{-08}$ & -0.59 & $9.07 \times 10^{-06}$ \\
\hline M482T134 & 1.34 & $8.26 \times 10^{-06}$ & -0.64 & $5.52 \times 10^{-07}$ \\
\hline M483T134 & 1.42 & $1.24 \times 10^{-05}$ & -0.56 & $3.68 \times 10^{-05}$ \\
\hline M489T128 & 1.59 & $2.06 \times 10^{-06}$ & -0.65 & $2.69 \times 10^{-07}$ \\
\hline M623T115 & 1.65 & $5.46 \times 10^{-08}$ & -0.50 & $3.80 \times 10^{-0}$ \\
\hline M631T132 & 1.57 & $6.29 \times 10^{-08}$ & -0.58 & $1.50 \times 10^{-0}$ \\
\hline M658T131 & 1.49 & $2.33 \times 10^{-08}$ & -0.65 & $3.25 \times 10^{-0}$ \\
\hline M672T472 & 3.90 & $1.85 \times 10^{-06}$ & 0.50 & $4.17 \times 10^{-0}$ \\
\hline M703T130_1 & 1.66 & $8.86 \times 10^{-10}$ & -0.54 & $8.58 \times 10^{-0}$ \\
\hline M704T131 & 1.56 & $4.21 \times 10^{-05}$ & -0.52 & $1.93 \times 10^{-0}$ \\
\hline M757T668 & 3.01 & $1.35 \times 10^{-06}$ & 0.51 & $2.51 \times 10^{-04}$ \\
\hline M777T418 & 3.89 & $2.64 \times 10^{-08}$ & 0.51 & $2.69 \times 10^{-04}$ \\
\hline M801T471 & 3.34 & $4.14 \times 10^{-07}$ & 0.48 & $7.36 \times 10^{-04}$ \\
\hline M819T421 & 3.56 & $1.66 \times 10^{-09}$ & 0.50 & $3.93 \times 10^{-04}$ \\
\hline M849T462 & 4.12 & $4.70 \times 10^{-11}$ & 0.51 & $2.28 \times 10^{-0}$ \\
\hline M865T412 & 3.04 & $6.13 \times 10^{-07}$ & 0.47 & $9.55 \times 10^{-6}$ \\
\hline M879T417 & 3.09 & $3.64 \times 10^{-07}$ & 0.47 & $9.91 \times 10^{-0}$ \\
\hline M923T419 & 3.12 & $1.89 \times 10^{-07}$ & 0.49 & $5.58 \times 10^{-0}$ \\
\hline
\end{tabular}

Cor, coefficient of association; FC, fold-change; P-value1, P-value of FC; P-value2, P-value of cor.

The mean value of the low-level samples in the close-knit area was $14.5 \mu \mathrm{mol} / 1$, compared with the high-level samples (17.0 $\mu \mathrm{mol} / \mathrm{l})$. In general, there may be an overlap between classes owing to the subjective classification thresholds of serum homocysteine, which was set at $15.0 \mu \mathrm{mol} / 1$.

The metabolic pathways that were significantly enriched for the aforementioned 77 compounds matched in Matlin database were analyzed and two pathways were identified (Table V; FDR $<0.05)$. The first enriched pathway was arachidonic acid metabolism $\left(\mathrm{FDR}=8.01 \times 10^{-8}\right)$, which serves an important role in vascular homeostasis; a total of 17 compounds that were
Table V. Metabolic pathway enrichment analysis.

\begin{tabular}{lcccc}
\hline Pathway name & Total & Hits & P-value & FDR \\
\hline $\begin{array}{l}\text { Arachidonic acid } \\
\text { metabolism }\end{array}$ & 62 & 17 & $1.00 \times 10^{-9}$ & $8.01 \times 10^{-8}$ \\
$\begin{array}{l}\text { Linoleic acid } \\
\text { metabolism }\end{array}$ & 15 & 4 & $4.29 \times 10^{-4}$ & $1.72 \times 10^{-2}$ \\
\hline \begin{tabular}{l} 
FDR, false discovery rate. \\
\hline
\end{tabular} & & & & \\
\hline
\end{tabular}

Table VI. Details of the 17 compounds in arachidonic acid metabolism pathway.

\begin{tabular}{|c|c|c|}
\hline Feature & KEGG ID & Name \\
\hline M321T139 & $\mathrm{C} 04742$ & 15(S)-HETE \\
\hline M321T139 & $\mathrm{C} 14770$ & 11,12-EET \\
\hline M321T139 & C14771 & $\begin{array}{l}\text { 14,15-epoxy-5,8,11-eicosatrienoic } \\
\text { acid }\end{array}$ \\
\hline M321T139 & C14778 & 16(R)-HETE \\
\hline M321T139 & C14749 & 19(S)-HETE \\
\hline M321T139 & C14748 & 20-HETE \\
\hline M321T139 & C14768 & $\begin{array}{l}5,6 \text {-epoxy-8,11,14-eicosatrienoic } \\
\text { acid }\end{array}$ \\
\hline M321T139 & $\mathrm{C} 04805$ & 5-HETE \\
\hline M321T139 & C14769 & 8,9-EET \\
\hline M367T135 & C05961 & 6-keto-prostaglandin F1 $\alpha$ \\
\hline M305T140 & $\mathrm{C} 00219$ & Arachidonic acid \\
\hline M335T120 & C05958 & $\Delta$ 12-Prostaglandin $\mathrm{J} 2$ \\
\hline M335T120 & C05953 & Prostaglandin A2 \\
\hline M335T120 & C05954 & Prostaglandin B2 \\
\hline M335T120 & $\mathrm{C} 05957$ & Prostaglandin $\mathrm{J} 2$ \\
\hline M335T120 & $\mathrm{C} 05955$ & Prostaglandin $\mathrm{C} 2$ \\
\hline M819T421 & $\mathrm{C} 00157$ & Phosphatidylcholine \\
\hline
\end{tabular}

EET, epoxyeicosatrienoic acid; HETE, hydroxyeicosatetraenoic acid.

Table VII. Details of the 4 compounds in linoleic acid metabolism pathway.

\begin{tabular}{lll}
\hline Feature & KEGG ID & \multicolumn{1}{c}{ Name } \\
\hline M819T421 & C00157 & Phosphatidylcholine \\
M313T131 & C04717 & 13-L-Hydroperoxylinoleic acid \\
M313T131 & C07354 & (7S,8S)-DiHODE \\
M313T131 & C14831 & 8(R)-Hydroperoxylinoleic acid
\end{tabular}

matched in the Matlin database were revealed to be involved in this pathway (Fig. 3A; Table VI). The second pathway was linoleic acid metabolism $\left(\mathrm{FDR}=1.72 \times 10^{-2}\right)$, and a total of 4 compounds that were matched in the Matlin database were involved in this pathway (Fig. 3B; Table VII). However, 


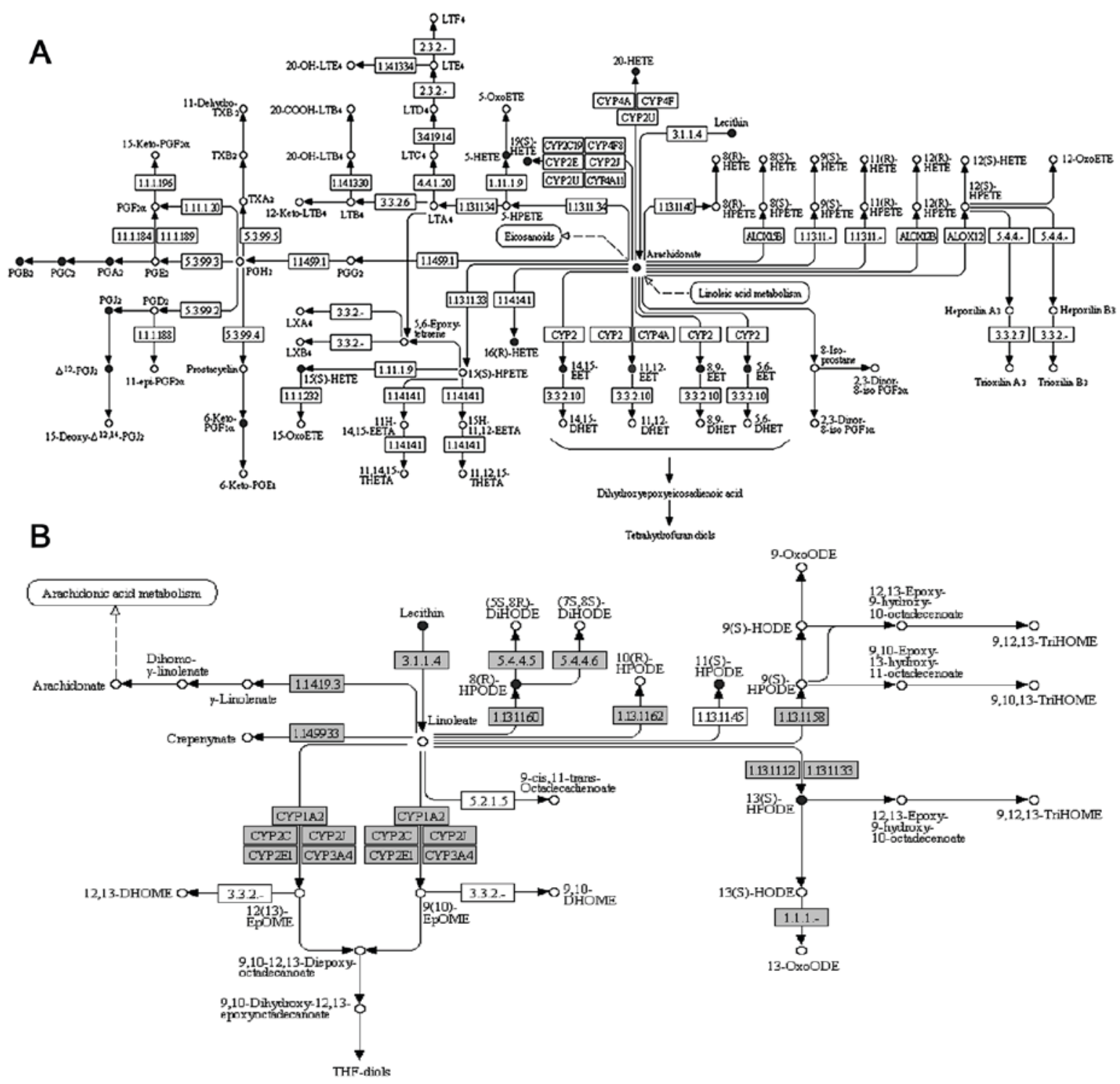

Figure 3. Arachidonic acid and linoleic acid metabolism signaling pathways. (A) The 17 compounds that were matched in Metlin database are annotated (filled black circles) in the arachidonic acid pathway. (B) The 4 compounds that were matched in Metlin database are annotated (filled black circles) in the linoleic acid metabolism signaling pathway.

a 'one-to-many' type of relationship was noted between the accurate mass and the identified compounds in the Matlin database.M321T139, for example, matched 9 compounds in the Matlin database, and all of the 9 compounds were annotated in the arachidonic acid metabolism pathway. This suggests that at least one 1 of the 9 compounds was abnormally metabolized in the arachidonic acid pathway. In this case, the false-positive rate of the pathway enrichment analysis may have been magnified. In order to obtain more reliable results in the pathway analysis, the one-to-many relationships between the accurate mass and the compounds were eliminated in the two pathways revealed to be significant, and only one compound was retained randomly in each one-to-many relationships. Regardless of which one compound was retained in the one-to-many relationships, there was no effect on the significance in the MetaboAnalyst 3.0 analysis. Subsequently, metabolic pathway enrichment was reanalyzed using MetaboAnalyst 3.0, and it was revealed that the arachidonic acid metabolism $(\mathrm{FDR}=0.02)$ and linoleic acid metabolism (FDR=0.12) remained the top two pathways significantly enriched with the 21 retained compounds.

\section{Discussion}

Results from the present study demonstrated a separation between the high- and the low-serum homocysteine groups using the correlated features detected in $\operatorname{ESI}(+)$ and ESI(-) modes. Furthermore, metabolic pathway analysis revealed that arachidonic acid metabolism and linoleic acid metabolism were significantly enriched for the compounds matched in the Matlin database. These results indicated that serum homocysteine levels may affect arachidonic acid and linoleic acid metabolism, and may subsequently contribute to the development of vascular diseases. Homocysteine was previously reported to have the potential to disrupt arachidonic acid metabolism through DNA demethylation in vitro (27).

A number of previous studies have suggested that arachidonic acid metabolism served an important role in vascular 
diseases (28-30). Arachidonic acid is a free fatty acid derived from membrane phospholipids (31), which is catalyzed by phospholipase A2 and metabolized into hundreds of metabolites by three pathways: The cyclooxygenase (COX), lipoxygenase (LOX) and cytochrome P450 (CYP450) pathways (32). The various arachidonic acid metabolites serve different roles in vascular diseases pathogenesis, and a total of 17 compounds annotated in arachidonic acid metabolism pathway maybe divided into three main groups: Prostaglandins, hydroxyeicosatetraenoic acids (HETEs) and epoxyeicosatrienoic acids (EETs). Prostaglandins are crucial bioactive molecules that are derived from the COX pathway and subsequent prostaglandin synthesis, and have been implicated in inflammation $(33,34)$. The HETEs are metabolites in the LOX pathway, and have been implicated in numerous biological processes, such as angiogenesis (35) and platelet activation (36). The EETs are metabolites in the CYP450 pathway, and may act as antihypertensive and antiatherosclerotic (37) mediators for vasculature. Therefore, an imbalanced arachidonic acid metabolism in vascular may lead to an impairment in vascular homeostasis and the subsequent development of vascular disease.

In conclusion, the present study provided novel insights into the effects of homocysteine on metabolic alterations in human serum. The results suggested that arachidonic acid and linoleic acid metabolic pathways may be involved in homocysteine-induced vascular disease. These data revealed a novel effect of homocysteine in vascular disease and may have clinical significance for the treatment of these diseases.

\section{Acknowledgements}

Not applicable.

\section{Funding}

This study was supported by The National High Technology Research and Development Program 863 (grant no. 2011AA02A111).

\section{Availability of data and materials}

The datasets used during the present study are available from the corresponding author on reasonable request.

\section{Authors' contributions}

The work presented here was performed in collaboration between all authors. HL, YT and XJ defined the research theme. BL, GG, WZ, BL and CY designed the methods and experiments, performed the laboratory experiments and analyzed the data. BL and WZ wrote the manuscript. All authors have contributed to, read and approved the manuscript.

\section{Ethics approval and consent to participate}

The present study was approved by the Ethics Committee of the Fourth Affiliated Hospital of Harbin Medical University (Harbin, China; 2018-SCILLSC-02). All participating subjects were informed of their rights and written informed consent was obtained.

\section{Consent for publication}

Not applicable.

\section{Competing interests}

The authors declare that they have no competing interests.

\section{References}

1. McCully KS: Vascular pathology of homocysteinemia: Implications for the pathogenesis of arteriosclerosis. Am J Pathol 56: 111-128, 1969.

2. Moll S: Plasma homocysteine levels and mortality in patients with coronary artery disease. N Engl J Med 337: 1631-1632; author reply 1632-1633, 1997

3. Soinio M, Marniemi J, Laakso M, Lehto $S$ and Rönnemaa T: Elevated plasma homocysteine level is an independent predictor of coronary heart disease events in patients with type 2 diabetes mellitus. Ann Intern Med 140: 94-100, 2004.

4. Arnesen E, Refsum H, Bønaa KH, Ueland PM, Førde OH and Nordrehaug JE: Serum total homocysteine and coronary heart disease. Int J Epidemiol 24: 704-709, 1995.

5. Cheng SW, Ting AC and Wong J: Fasting total plasma homocysteine and atherosclerotic peripheral vascular disease. Ann Vasc Surg 11: 217-223, 1997.

6. Graham IM, Daly LE, Refsum HM, Robinson K, Brattström LE, Ueland PM, Palma-Reis RJ, Boers GH, Sheahan RG, Israelsson B, et al: Plasma homocysteine as a risk factor for vascular disease. The European Concerted Action Project. JAMA 277: 1775-1781, 1997.

7. Perry IJ, Refsum H, Morris RW, Ebrahim SB, Ueland PM and Shaper AG: Prospective study of serum total homocysteine concentration and risk of stroke in middle-aged British men. Lancet 346: 1395-1398, 1995.

8. Towfighi A, Saver JL, Engelhardt R and Ovbiagele B: Factors associated with the steep increase in late-midlife stroke occurrence among US men. J Stroke Cerebrovasc Dis 17: 165-168, 2008.

9. Homocysteine Studies Collaboration: Homocysteine and risk of ischemic heart disease and stroke: A meta-analysis. JAMA 288: 2015-2022, 2002.

10. Boushey CJ, Beresford SA, Omenn GS and Motulsky AG: A quantitative assessment of plasma homocysteine as a risk factor for vascular disease. Probable benefits of increasing folic acid intakes. JAMA 274: 1049-1057, 1995.

11. Selhub J: Homocysteine metabolism. Annu Rev Nutr 19: 217-246, 1999.

12. McCully KS: Homocysteine and the pathogenesis of atherosclerosis. Expert Rev Clin Pharmacol 8: 211-219, 2015.

13. Nicholson JK, Lindon JC and Holmes E: 'Metabonomics': Understanding the metabolic responses of living systems to pathophysiological stimuli via multivariate statistical analysis of biological NMR spectroscopic data. Xenobiotica 29: 1181-1189, 1999.

14. Wikoff WR, Gangoiti JA, Barshop BA and Siuzdak G: Metabolomics identifies perturbations in human disorders of propionate metabolism. Clin Chem 53: 2169-2176, 2007.

15. Xue R, Lin Z, Deng C, Dong L, Liu T, Wang J and Shen X: A serum metabolomic investigation on hepatocellular carcinoma patients by chemical derivatization followed by gas chromatography/mass spectrometry. Rapid Commun Mass Spectrom 22: 3061-3068, 2008.

16. Bogdanov M, Matson WR, Wang L, Matson $T$, Saunders-Pullman R, Bressman SS and Flint Beal M: Metabolomic profiling to develop blood biomarkers for Parkinson's disease. Brain 131: 389-396, 2008.

17. Nichols on JK, Connelly J, Lindon JC and Holmes E: Metabonomics: A platform for studying drug toxicity and gene function. Nat Rev Drug Discov 1: 153-161, 2002.

18. Want EJ, Nordström A, Morita H and Siuzdak G: From exogenous to endogenous: The inevitable imprint of mass spectrometry in metabolomics. J Proteome Res 6: 459-468, 2007.

19. Joint Committee for Developing Chinese guidelines on Prevention and Treatment of Dyslipidemia in Adults. Chinese guidelines on prevention and treatment of dyslipidemia in adults. Zhonghua Xin Xue Guan Bing Za Zhi 35: 390-419, 2007 (In Chinese). 
20. Chen C and Lu FC; Department of Disease Control Ministry of Health, PR China: The guidelines for prevention and control of overweight and obesity in Chinese adults. Biomed Environ Sci 17 (Suppl): S1-S36, 2004

21. Guo H, Chi J, Xing Y and Wang P: Influence of folic acid on plasma homocysteine levels \& arterial endothelial function in patients with unstable angina. Indian J Med Res 129: 279-284, 2009.

22. Xu J, Tian YP, Chen YH, Zhang RP, YANG F, Song YM and Zeper A: Plasma preparation method for metabollomic analysis based on rapid resolution liquid chromatography-mass spectrometry. Chin J Anal Chem 39: 1793-1797, 2011.

23. Smith CA, Want EJ, O'Maille G, Abagyan R and Siuzdak G: XCMS: Processing mass spectrometry data for metabolite profiling using nonlinear peak alignment, matching, and identification. Anal Chem 78: 779-787, 2006.

24. Xia J, Mandal R, Sinelnikov IV, Broadhurst D and Wishart DS MetaboAnalyst 2.0-a comprehensive server for metabolomic data analysis. Nucleic Acids Res 40 (Web Server Issue): W127-W133, 2012.

25. Xia J, Sinelnikov IV, Han B and Wishart DS: MetaboAnalyst 3.0-making metabolomics more meaningful. Nucleic Acids Res 43 (W1): W251-W257, 2015.

26. Xia J and Wishart DS: MetPA: A web-based metabolomics tool for pathway analysis and visualization. Bioinformatics 26 : 2342-2344, 2010.

27. Xue SS, He JL, Zhang X, Liu YJ, Xue FX, Wang CJ, Ai D and Zhu Y: Metabolomic analysis revealed the role of DNA methylation in the balance of arachidonic acid metabolism and endothelial activation. Biochim Biophys Acta 1851: 1317-1326, 2015.

28. Capdevila JH, Falck JR and Harris RC: Cytochrome P450 and arachidonic acid bioactivation. Molecular and functional properties of the arachidonate monooxygenase. J Lipid Res 41: 163-181, 2000.

29. Wang Y,Wei X, Xiao X, Hui R, Card JW, Carey MA, Wang DW and Zeldin DC: Arachidonic acid epoxygenase metabolites stimulate endothelial cell growth and angiogenesis via mitogen-activated protein kinase and phosphatidylinositol 3-kinase/Akt signaling pathways. J Pharmacol Exp Ther 314: 522-532, 2005.
30. Xiao B, Li X, Yan J, Yu X, Yang G, Xiao X, Voltz JW, Zeldin DC and Wang DW: Overexpression of cytochrome P450 epoxygenases prevents development of hypertension in spontaneously hypertensive rats by enhancing atrial natriuretic peptide. J Pharmacol Exp Ther 334: 784-794, 2010.

31. Smith GI, Atherton P, Reeds DN, Mohammed BS, Rankin D, Rennie MJ and Mittendorfer B: Omega-3 polyunsaturated fatty acids augment the muscle protein anabolic response to hyperaminoacidemia-hyperinsulinemiain healthy young and middle-aged men and women. Clin Sci (Lond) 121: 267-278, 2011.

32. Imig JD and Hammock BD: Soluble epoxide hydrolase as a therapeutic target for cardiovascular diseases. Nat Rev Drug Discov 8: 794-805, 2009

33. Nishimura $M$, Hirai A, Omura $M$, Tamura $Y$ and Yoshida S: Arachidonic acid metabolites by cytochrome P-450 dependent monooxygenase pathway in bovine adrenal fasciculata cells. Prostaglandins 38: 413-430, 1989.

34. Capdevila J, Marnett LJ, Chacos N, Prough RA and Estabrook RW: Cytochrome P-450-dependent oxygenation of arachidonic acid to hydroxyicosatetraenoic acids. Proc Natl Acad Sci USA 79: 767-770, 1982.

35. Ma C, Wang Y, Shen T, Zhang C, Ma J, Zhang L, Liu F and Zhu D: Placenta growth factor mediates angiogenesis in hypoxic pulmonary hypertension. Prostaglandins Leukot Essent Fatty Acids 89: 159-168, 2013.

36. Blazevic T, Schwaiberger AV, Schreiner CE, Schachner D, Schaible AM, Grojer CS, Atanasov AG, Werz O, Dirsch VM and Heiss EH: 12/15-lipoxygenase contributes to platelet-derived growth factor-induced activation of signal transducer and activator of transcription 3. J Biol Chem 288: 35592-35603, 2013.

37. Bellien $\mathrm{J}$ and Joannides R: Epoxyeicosatrienoic acid pathway in human health and diseases. J Cardiovasc Pharmacol 61: 188-196, 2013.

This work is licensed under a Creative Commons Attribution-NonCommercial-NoDerivatives 4.0 International (CC BY-NC-ND 4.0) License. 